\title{
Chemokine receptor CCR7 enhances intrahepatic and lymphatic dissemination of human hepatocellular cancer
}

\author{
CARL C. SCHIMANSKI ${ }^{1}$, RÜDIGER BAHRE ${ }^{1}$, INES GOCKEL ${ }^{2}$, THEODOR JUNGINGER ${ }^{2}$, \\ NEKTARIA SIMIANTONAKI ${ }^{3}$, STEFAN BIESTERFELD ${ }^{3}$, TOBIAS ACHENBACH ${ }^{4}$, \\ THOMAS WEHLER ${ }^{5}$, PETER R. GALLE ${ }^{1}$ and MARKUS MOEHLER ${ }^{1}$ \\ ${ }^{1}$ First Department of Internal Medicine, ${ }^{2}$ Institute of Surgery, ${ }^{3}$ Institute of Pathology, ${ }^{4}$ Department of Radiology, \\ ${ }^{5}$ Third Department of Internal Medicine, Johannes Gutenberg University of Mainz, Mainz, Germany
}

Received March 13, 2006; Accepted April 12, 2006

\begin{abstract}
Despite many pathophysiological analyses, the process of tumor dissemination of hepatocellular carcinoma (HCC) remains vague. In diverse tumor entities, expression of the chemokine receptor, CCR7, has been linked to tumor dissemination and poor prognosis. Therefore, we evaluated, whether CCR7 exerts similar effects in human HCC. CCR7 expression analysis was performed in vitro on human hepatoma cell lines (Huh7, Hep3B, wt HepG2, p53 dominant negative transfected HepG2). In addition, CCR7 expression was evaluated in 39 patients with hepatocellular cancer and correlated with both, tumor and patients characteristics. Human hepatocellular carcinoma samples and hepatoma cell lines displayed variable intensities of CCR7 expression. In patients, CCR7 expression was significantly associated with progressed local tumors $(\mathrm{P}=0.02)$ and lymphatic metastasis $(\mathrm{P}=0.02)$. Strong expression of CCR7 promotes intrahepatic and lymphatic HCC dissemination.
\end{abstract}

\section{Introduction}

Hepatocellular carcinoma (HCC) is one of the most common cancer entities with an annual incidence of more than 500,000 cases and raising incidence rates in Western countries during the $1990 \mathrm{~s}(1,2)$. HCC is often related to liver cirrhosis commonly resulting from chronic inflammatory liver disease, such as chronic viral hepatitis or autoimmune hepatitis/ cholangitis, but also from other diseases, such as chronic alcohol abuse or consumption of mycotoxin and aflatoxin B1 (3). Hepatocarcinogenesis is regarded as a slow process in

Correspondence to: Dr Carl C. Schimanski, First Department of Internal Medicine, Johannes Gutenberg University of Mainz, Langenbeckstrasse 1, D-55101 Mainz, Germany

E-mail: dr_schimanski@yahoo.de

Abbreviations: bp, base pair; HCC, hepatocellular carcinoma; CCR7, chemokine receptor 7

Key words: chemokine, cancer, liver, hepatocellular, metastasis which diverse genomic alterations accumulate, modifying the phenotype of hepatocytes and leading to monoclonal and dysplastic hepatocyte populations (4). As progress of dysplastic hepatocytes to hepatocellular carcinoma might occur simultaneously in different foci or nodules, variable genomic alterations can be found within the same liver indicating a genetic heterogeneity of lesions (5). Diverse molecular determinants including mutations and loss of heterozygosity $(\mathrm{LOH})$ in certain tumor-suppressor genes and oncogenes have been summarized in the pathway of molecular pathogenesis of human hepatocellular carcinoma $(4,5)$. Nonetheless, additional pathogenic alterations are considered to instrumentally mediate the progression and dissemination of human HCC.

Diverse results suggest that chemokine receptors may direct lymphatic and hematogenous spreading and may furthermore influence the sites of metastatic growth of different tumors (6). Chemokines and their G-protein-coupled receptors mediate pro- and anti-inflammatory responses (7). CCR7, the receptor for the chemokine, CCL21, is expressed on naïve T-cells, on memory $\mathrm{T}$ cells, B cells and mature dendritic cells and is considered to play an important role in lymphocyte cell trafficking and homing to lymph nodes $(8,9)$. Most recently, CCR7 has shifted into focus as it might play an important role in tumor spreading. CCR7 expression positively correlated with lymphatic metastasis and poor prognosis in rectal, gastric and esophageal squamous cell carcinoma (10-12). Supporting data from in vivo models underlined the key roles of CCR7 for migration (13).

However, no data are available on the CCR7 expression in human hepatocellular carcinoma and its impact on disease progression and prognosis. Therefore, we analyzed the CCR7 expression in hepatoma cell lines and hepatocellular carcinoma specimens and correlated the results with the patients' clinicopathological parameters and survival.

\section{Materials and methods}

Cell culture. The human hepatoma cell lines, Huh-7, Hep3B, HepG2 and p53 dominant negative transfected HepG2, were cultured in DMEM (Invitrogen, Germany) supplemented with $10 \%$ FCS, 100 units $/ \mathrm{ml}$ penicillin, $100 \mu \mathrm{g} / \mathrm{ml}$ streptomycin (Cambrex, Germany) and $1 \mathrm{mM} \mathrm{L-glutamine} \mathrm{(Invitrogen).} \mathrm{p53}$ dominant negative transfected HepG2 were kindly provided by 


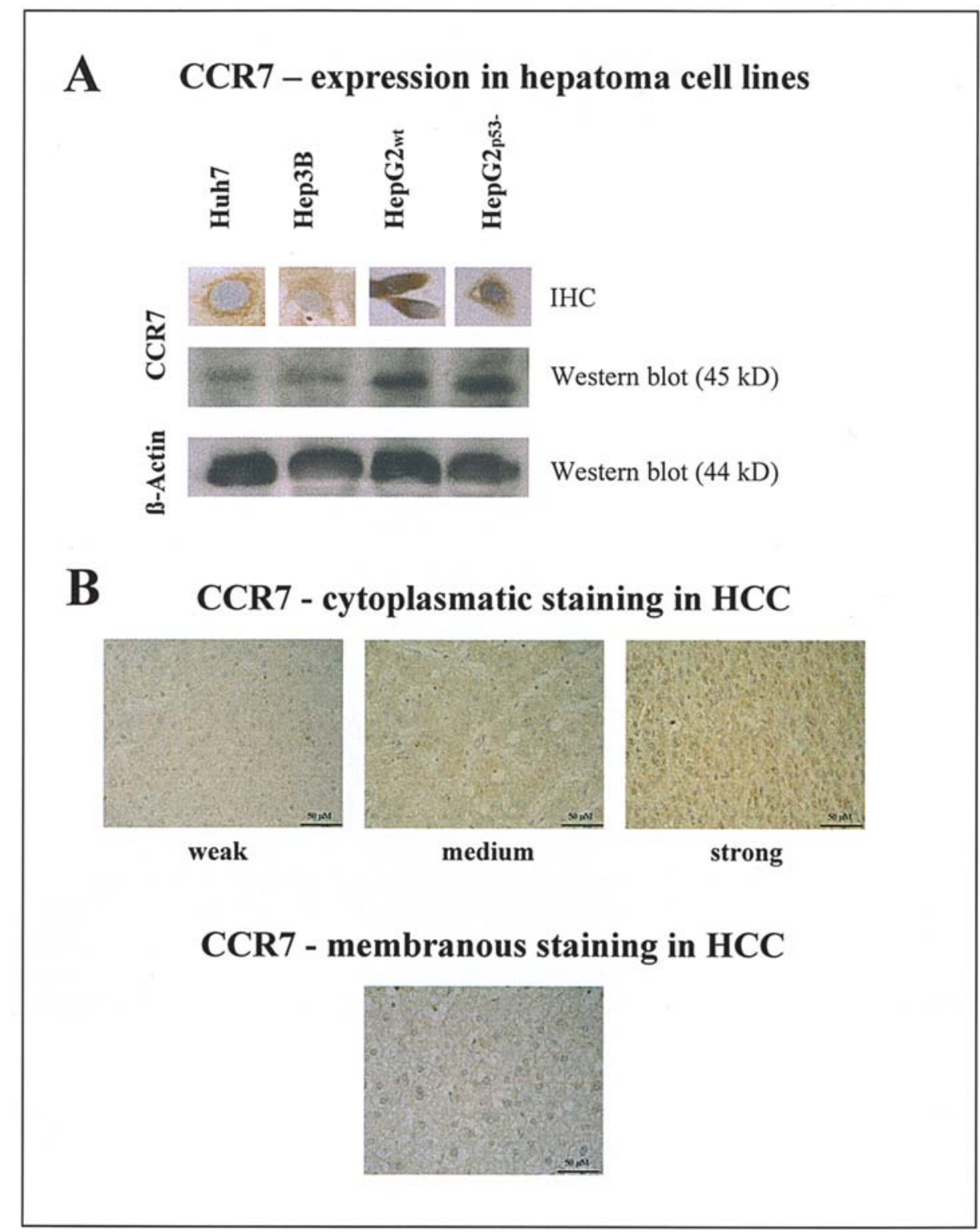

Figure 1. (A) Expression and immunohistochemical staining of CCR7 in diverse human hepatoma cell lines. Huh7 and Hep3B cells revealed medium CCR7 expression, whereas HepG2 cell lines depicted strong CCR7 staining, independent from p53 status. (B) Intensity of CCR7 expression in different hepatocellular carcinomas. The respective cytoplasmatic expression grades of CCR7 (weak, medium, strong) as well as a sample of membranous CCR7 expression.

M. Schuler (Third Department of Internal Medicine, Johannes Gutenberg University, Mainz, Germany).

Western blot analysis. Huh-7, Hep3B, HepG2 and p53 dominant negative transfected HepG2 cells were washed with PBS and lysed in $0.5 \%$ NP-40 solution. Protein $(100 \mu \mathrm{g})$ was loaded on an $10 \%$ SDS-PAGE gel. The gel was transferred onto a PVDF membrane following seperation. The respective proteins were detected with anti-CCR-7 (1:500, CIO131, Capralogics, USA; 1:1000 donkey anti-goat IgG 2nd antibody SC-2020 by Santa Cruz) and anti-Actin (1:1000, A2066, Sigma, Germany; 1:1000 goat anti-rabbit IgG 2nd antibody 170-6515 by BioRad, USA) and were visualized using an ECL Western blotting analysis system (Amersham Biosciences, USA).

Tissue samples. Hepatocellular carcinoma tissue samples were obtained from 39 patients undergoing liver biopsy, hemihepatectomy or orthotopic liver transplantation for hepatocellular carcinoma at the University of Mainz. The morphological classification of the carcinomas was conducted according to World Health Organization (WHO) specifications. Patients were followed up on a regular basis depending on the procedure performed.

Immunohistochemical staining. The avidin-biotin-complex method was used to detect CCR-7 (anti-CCR-7, dilution 1:250; Capralogics). Formalin-fixed and paraffin-embedded tissue were deparaffinised and subsequently microwaved in EDTA buffer. After pre-incubation with hydrogen peroxide, avidin/biotin blocking kit (Vector Laboratories Inc., USA) and rabbit serum (Vector Laboratories Inc.), the primary antibodies were applied for $1 \mathrm{~h}$ at room temperature. After incubation with the secondary antibody (rabbit anti-goat biotinylated; dilution 1:200, Vector Laboratories Inc.), the avidinbiotin complex was added and the enzyme activity visualized 
with diaminobenzidine. Counterstaining was performed with haematoxylin. For negative controls, only the secondary antibody was used. A negative control was performed for every hepatocellular carcinoma sample $(n=39)$. For positive controls, formalin-fixed and paraffin-embedded tissue samples of the human spleen were applied.

Evaluation of immunostaining. Immunostaining was evaluated by three independent authors (C.C.S., R.B., N.S.) blinded to patient outcome and all clinicopathologic findings. The immunohistochemical staining was analyzed according to a scoring method that we have previously validated and described (10): the tumors were classified into four groups based on the homogenous staining intensity: 0 , absent; 1 , weak; 2 , intermediate; 3 , strong staining. In the case of heterogeneous staining within the same sample, the respective, 0.5 points higher score was chosen, if more than $50 \%$ of cells revealed the higher staining intensity. If the evaluations did not agree, the specimens were reevaluated and then classified according to the assessment given most frequently by the observers.

Statistics. The association of staining intensity with clinicopathological patterns was assessed with the $\chi^{2}$ test and with the unpaired Student t-test, when appropriate. Survival rates were visualized applying the Kaplan-Meier curves, and P-values were determined by the log-rank test. $\mathrm{P}<0.05$ was considered significant and $\mathrm{P}<0.001$ was highly significant in all statistical analyses.

\section{Results}

CCR7 expression in hepatoma cell lines. CCR7 expression of human hepatoma cell lines revealed varying expression intensities as depicted by Fig. 1A. Notably, p53 did not impact on CCR7 expression. CCR7-immunostaining results correlated with the respective Western blot analysis.

Tumor characteristics and patient profiles. The group of patients represent the typical characteristics of hepatocellular cancer in industrialized countries, except for a lower percentage of liver cirrhosis associated-HCC and female patients. The increased survival times result from the low percentage of cirrhosis among our patients. Patients characteristics are depicted in Table I.

Immunohistochemical staining of CCR7 in hepatocellular carcinoma. The staining for CCR7 revealed predominantly a cytoplasmatic and, in a few specimens, an additional weak membranous location of CCR7 (Fig. 1B). Nuclear staining of CCR7 was not observed. CCR7 expression was also found in $100 \%(39 / 39)$ of all hepatocellular cancer specimens and varied between weak $(8 \%)$, intermediate $(49 \%)$ and strong $(43 \%)$.

Negative controls of human hepatocellular cancer remained negative for every tissue sample $(n=39$, data not shown). Splenic lymphocytes revealed strong CCR7 expression, matching the strong CCR7 expression of human hepatocellular cancer tissue or cell lines. Similarly, inflammatory infiltrates of the liver depicted strong CCR7 expression.

Relevance of CCR7 expression in hepatocellular carcinoma. Strong CCR7 expression also correlated with local progression
Table I. Patient and tumor characteristics.

\section{Patient characteristics}

Total no.

Median age (years)

Gender

Female

Male

$35(90 \%)$

T-status

1

2

$8(20 \%)$

$10(26 \%)$

3

$16(41 \%)$

4

$5(13 \%)$

$\mathrm{N}$-status

0

$24(62 \%)$

1

$10(26 \%)$

2

$4(10 \%)$

Unknown

M-status

0

$26(67 \%)$

1

Unknown

Grading

1

2

$25(64 \%)$

3

$11(28 \%)$

3-year survival

$54 \%$

Liver cirrhosis

Yes

$19(49 \%)$

No

$18(46 \%)$

Unknown

$25 \%)$

and proliferation of the primary tumor as indicated by the Tstatus (TNM classification; $\mathrm{P}=0.02$; Table II) and with lymph node involvement ( $\mathrm{N}$-status; $\mathrm{P}=0.02)$, but not with distant metastases (M-status; $\mathrm{P}=0.3$ ). $\mathrm{CCR} 7$ expression was neither associated with gender, age or grading $(G)$. In the group of patients with underlying cirrhosis, no correlation between etiology of cirrhosis or Child-Phough classification and the CCR7 expression level was observed. CCR7 expression did not correlate with the 3-year survival.

\section{Discussion}

So far, no data have been available on the CCR7 expression in human hepatocellular carcinoma. In diverse tumor entities, expression of CCR7 has been linked to tumor dissemination and poor prognosis. Therefore, we analyzed the CCR7 expression profile in a series of human hepatoma cell lines and hepatocellular carcinoma patients. 
Table II. Patient and tumor characteristics dependent on intensity of CCR7 expression.

\begin{tabular}{|c|c|c|c|c|}
\hline & \multicolumn{3}{|c|}{ CCR7 expression } & \multirow[t]{2}{*}{ Statistics } \\
\hline & Weak (1) & ) Intermediate (2) & Strong (3) & \\
\hline Total no. & $3(8 \%)$ & $19(49 \%)$ & $17(43 \%)$ & \\
\hline $\begin{array}{l}\text { Average } \\
\text { age (years) }\end{array}$ & & 63.8 & 57.2 & $\mathrm{~ns}$ \\
\hline \multicolumn{5}{|l|}{ Gender } \\
\hline Female & & 2 & 2 & ns \\
\hline Male & & 20 & 15 & \\
\hline \multicolumn{5}{|l|}{ T-status } \\
\hline $1+2$ & & 14 & 4 & $\mathrm{P}=0.02$ \\
\hline $3+4$ & & 8 & 13 & \\
\hline \multicolumn{5}{|l|}{ N-status ${ }^{\mathrm{a}}$} \\
\hline 0 & & 17 & 7 & $\mathrm{P}=0.02$ \\
\hline+ & & 4 & 10 & \\
\hline \multicolumn{5}{|l|}{ M-status ${ }^{\mathrm{a}}$} \\
\hline 0 & & 16 & 10 & ns \\
\hline+ & & 5 & 7 & \\
\hline \multicolumn{5}{|l|}{ Grading } \\
\hline $1+2$ & & 16 & 12 & ns \\
\hline $3+4$ & & 6 & 5 & \\
\hline $\begin{array}{l}\text { 3-year } \\
\text { survival }\end{array}$ & & $53 \%$ & $63 \%$ & ns \\
\hline \multicolumn{5}{|l|}{$\begin{array}{l}\text { Liver } \\
\text { cirrhosis }^{b}\end{array}$} \\
\hline Yes & & 12 & 7 & ns \\
\hline No & & 8 & 10 & \\
\hline
\end{tabular}

${ }^{\mathrm{a}} \mathrm{N}$ and $\mathrm{M}$ status could not be obtained from one patient. ${ }^{\mathrm{b}}$ Liver status could not be obtained from two patients.

All human hepatoma cell lines investigated depicted different intensities of a predominantly cytoplasmatic and less membraneous CCR7 expression.

Immunohistochemical staining of human hepatocellular carcinoma specimens displayed predominantly cytoplasmatic and, in fewer cases, membranous CCR7 expression with variable intensities, matching the observations made in human hepatoma cell lines.

In our patients, strong CCR7 expression was significantly associated with locally progressed tumors and lymph node metastases. As progressed T-status in HCC is defined as intrahepatic growth and spread of the respective hepatocellular cancer, our results also imply a substantial influence of CCR7 on intrahepatic dissemination of hepatocellular carcinoma. In summary, our data are in line with results described for esophageal, gastric and rectal cancer (10-12).

The lungs, liver and lymph nodes are major target organs for metastases in men. Homing factors, influencing chemotaxis to target organs, have been proposed as major inductors of site-specific metastases, as the filter function of these organs not solely explains the growth of metastases (14-16). CCR7 mediated lymphatic dissemination has been compared to the homing of activated dendritic cells to CCL21 expressing lymph nodes via lymphatic vessels $(12,13,17,18)$. The theory of CCR7 co-mediated mechanism of lymphatic dissemination is underlined by data, reporting that CCR7 expression of melanoma cells increased metastases formation in regional lymph nodes of mice (19).

Certain chemokines have been proposed to distinctly contribute to tumor growth, dissemination and local immune scape $(20,21)$. Our results are in line with these data for human hepatocellular carcinoma. Strong CCR7 expression by hepatocellular carcinoma was significantly associated with intrahepatic and nodal dissemination. Thus, CCR7 plays a relevant role during hepatocellular carcinoma progression. Further efforts will be necessary to analyze CCR7-dependent pathways of nodal dissemination.

\section{References}

1. Parkin DM, Bray FI and Devesa SS: Cancer burden in the year 2000. The global picture. Eur J Cancer 37 (suppl 8): S4-S66, 2001 .

2. Wingo PA, Cardinez CJ, Landis SH, et al: Long-term trends in cancer mortality in the United States, 1930-1998. Cancer 97 (suppl 12): S3133-S3275, 2003.

3. Bosch FX, Ribes J and Borras J: Epidemiology of primary liver cancer. Semin Liver Dis 19: 271-285, 1999.

4. Thorgeirsson SS and Grisham JW: Molecular pathogenesis of human hepatocellular carcinoma. Nat Genet 31: 339-346, 2002.

5. Feitelson MA, Sun B, Satiroglu Tufan NL, Liu J, Pan J and Lian Z: Genetic mechanisms of hepatocarcinogenesis. Oncogene 21: 2593-2604, 2002.

6. Arya M, Patel HR and Williamson M: Chemokines: key players in cancer. Curr Med Res Opin 19: 557-564, 2003

7. Zlotnik A and Yoshie O: Chemokines: a new classification system and their role in immunity. Immunity 12: 121-127, 2000.

8. Dieu MC, Vanbervliet B, Vicari A, et al: Selective recruitment of immature and mature dendritic cells by distinct chemokines expressed in different anatomic sites. J Exp Med 188: 373-386, 1998.

9. Hirao M, Onai N, Hiroishi K, et al: CC chemokine receptor-7 on dendritic cells is induced after interaction with apoptotic tumor cells: critical role in migration from the tumor site to draining lymph nodes. Cancer Res 60: 2209-2217, 2000.

10. Schimanski CC, Schwald S, Simiantonaki N, et al: Effect of chemokine receptors CXCR4 and CCR7 on the metastatic behavior of human colorectal cancer. Clin Cancer Res 11: 1743-1750, 2005

11. Ding Y, Shimada Y, Maeda M, et al: Association of CC chemokine receptor 7 with lymph node metastasis of esophageal squamous cell carcinoma. Clin Cancer Res 9: 3406-3416, 2003.

12. Mashino K, Sadanaga N, Yamaguchi H, et al: Expression of chemokine receptor CCR7 is associated with lymph node metastasis of gastric carcinoma. Cancer Res 62: 2937-2941, 2002.

13. Saeki H, Moore AM, Brown MJ and Hwang ST: Cutting edge: secondary lymphoid-tissue chemokine (SLC) and CC chemokine receptor 7 (CCR7) participate in the emigration pathway of mature dendritic cells from the skin to regional lymph nodes. J Immunol 162: 2472-2475, 1999.

14. Stetler-Stevenson WG and Kleiner DE: Molecular biology of cancer: invasion and metastases. Lippincott \& Wilkins, pp123-136, 2001.

15. Weiss L, Grundmann E, Torhorst J, et al: Haematogenous metastatic patterns in colonic carcinoma: an analysis of 1541 necropsies. J Pathol 150: 195-203, 1986.

16. Nicolson GL: Paracrine and autocrine growth mechanisms in tumor metastasis to specific sites with particular emphasis on brain and lung metastasis. Cancer Metastasis Rev 12: 325-343, 1993. 
17. Henning G, Ohl L, Junt T, et al: CC chemokine receptor 7dependent and -independent pathways for lymphocyte homing: modulation by FTY720. J Exp Med 194: 1875-1881, 2001.

18. Okada T, Ngo VN, Ekland EH, et al: Chemokine requirements for B cell entry to lymph nodes and Peyer's patches. J Exp Med 196: 65-75, 2002.

19. Wiley HE, Gonzalez EB, Maki W, Wu MT and Hwang ST: Expression of $\mathrm{CC}$ chemokine receptor-7 and regional lymph node metastasis of B16 murine melanoma. J Natl Cancer Inst 93: 1638-1643, 2001.
20. Balkwill F and Mantovani A: Inflammation and cancer: back to Virchow? Lancet 357: 539-545, 2001.

21. Brigati C, Noonan DM, Albini A and Benelli R: Tumors and inflammatory infiltrates: friends or foes? Clin Exp Metastasis 19: 247-258, 2002. 